\title{
PERIODIC ORBITS OF HAMILTONIAN SYSTEMS: APPLICATIONS TO PERTURBED KEPLER PROBLEMS
}

\author{
JUAN L.G. GUIRAO ${ }^{1}$, JAUME LLIBRE ${ }^{2}$ AND JUAN A. VERA ${ }^{3}$
}

\begin{abstract}
We provide for a class of Hamiltonian systems in the actionangle variables sufficient conditions for showing the existence of periodic orbits. We expand this result to the study of the existence of periodic orbits of perturbed spatial Keplerian Hamiltonians with axial symmetry. Finally, we apply these general results for finding periodic orbits of the MateseWhitman Hamiltonian, of the spatial anisotropic Hamiltonian and of the spatial generalized van der Waals Hamiltonian.
\end{abstract}

\section{IntRoduCtion AND STATEMENT OF THE MAIN RESUlts}

We consider the following class of Hamiltonians in the action-angle variables

$$
\mathcal{H}\left(I_{1}, \ldots, I_{n}, \theta_{1}, \ldots, \theta_{n}\right)=\mathcal{H}_{0}\left(I_{1}\right)+\varepsilon \mathcal{H}_{1}\left(I_{1}, \ldots, I_{n}, \theta_{1}, \ldots, \theta_{n}\right),
$$

where $\varepsilon$ is a small parameter.

As usual the Poisson bracket of the functions $f\left(I_{1}, \ldots, I_{n}, \theta_{1}, \ldots, \theta_{n}\right)$ and $g\left(I_{1}, \ldots, I_{n}, \theta_{1}, \ldots, \theta_{n}\right)$ is

$$
\{f, g\}=\sum_{i=1}^{n}\left(\frac{\partial f}{\partial \theta_{i}} \frac{\partial g}{\partial I_{i}}-\frac{\partial f}{\partial I_{i}} \frac{\partial g}{\partial \theta_{i}}\right) .
$$

The next result provides sufficient conditions for computing periodic orbits of the Hamiltonian system associated to the Hamiltonian (1).

Theorem 1. We define

$$
\left\langle\mathcal{H}_{1}\right\rangle=\frac{1}{2 \pi} \int_{0}^{2 \pi} \mathcal{H}_{1}\left(I_{1}, \ldots, I_{n}, \theta_{1}, \ldots, \theta_{n}\right) d \theta_{1},
$$

and we consider the differential system

$$
\begin{aligned}
\frac{d I_{i}}{d \theta_{1}} & =\varepsilon \frac{\left\{I_{i},\left\langle\mathcal{H}_{1}\right\rangle\right\}}{\mathcal{H}_{0}^{\prime}\left(\mathcal{H}_{0}^{-1}\left(h^{*}\right)\right)}=\varepsilon f_{i-1}\left(I_{2}, \ldots, I_{n}, \theta_{2}, \ldots, \theta_{n}\right) \quad i=2, \ldots, n, \\
\frac{d \theta_{i}}{d \theta_{1}} & =\varepsilon \frac{\left\{\theta_{i},\left\langle\mathcal{H}_{1}\right\rangle\right\}}{\mathcal{H}_{0}^{\prime}\left(\mathcal{H}_{0}^{-1}\left(h^{*}\right)\right)}=\varepsilon f_{i+n-2}\left(I_{2}, \ldots, I_{n}, \theta_{2}, \ldots, \theta_{n}\right) \quad i=2, \ldots, n,
\end{aligned}
$$

Key words and phrases. Periodic orbits, perturbed Kepler problem, the MateseWhitman Hamiltonian, spatial anisotropic Kepler problem, spatial generalized van del Waals Hamiltonian.

2010 Mathematics Subject Classification. Primary: 70F05, 37C27, 37J30. Secondary: 70F15, 37J25. 
restricted to the energy level $\mathcal{H}=h^{*}$ with $h^{*} \in \mathbb{R}$. The value $h^{*}$ is such that the function $\mathcal{H}_{0}^{-1}$ in a neighborhood of $h^{*}$ is a diffeomorphism. System (2) is a Hamiltonian system with Hamiltonian $\varepsilon\left\langle\mathcal{H}_{1}\right\rangle$. If $\varepsilon \neq 0$ is sufficiently small then for every equilibrium point $p=\left(I_{2}^{0}, \ldots, I_{n}^{0}, \theta_{2}^{0}, \ldots, \theta_{n}^{0}\right)$ of system (2) satisfying that

$$
\operatorname{det}\left(\left.\frac{\partial\left(f_{1}, \ldots, f_{2 n-2}\right)}{\partial\left(I_{2}, \ldots, I_{n}, \theta_{2}, \ldots, \theta_{n}\right)}\right|_{\left(I_{2}, \ldots, I_{n}, \theta_{2}, \ldots, \theta_{n}\right)=\left(I_{2}^{0}, \ldots, I_{n}^{0}, \theta_{2}^{0}, \ldots, \theta_{n}^{0}\right)}\right) \neq 0,
$$

there exists a $2 \pi$-periodic solution $\gamma_{\varepsilon}\left(\theta_{1}\right)=\left(I_{1}\left(\theta_{1}, \varepsilon\right), \ldots, I_{n}\left(\theta_{1}, \varepsilon\right), \theta_{2}\left(\theta_{1}, \varepsilon\right), \ldots\right.$, $\left.\theta_{n}\left(\theta_{1}, \varepsilon\right)\right)$ of the Hamiltonian system associated to the Hamiltonian (1) taking as independent variable the angle $\theta_{1}$ such that $\gamma_{\varepsilon}(0) \rightarrow\left(\mathcal{H}_{0}^{-1}\left(h^{*}\right), I_{2}^{0}, \ldots, I_{n}^{0}, \theta_{2}^{0}\right.$, $\left.\ldots, \theta_{n}^{0}\right)$ when $\varepsilon \rightarrow 0$. The stability or instability of the periodic solution $\gamma_{\varepsilon}\left(\theta_{1}\right)$ is given by the stability or instability of the equilibrium point $p$ of system (2). In fact, the equilibrium point $p$ has the stability behavior of the Poincaré map associated to the periodic solution $\gamma_{\varepsilon}\left(\theta_{1}\right)$.

Now we clarify some of the notations used in the statement of Theorem 1. We have that the function $\mathcal{H}_{0}$ is only function of the variable $I_{1}$, i.e. $\mathcal{H}_{0}$ : $J \rightarrow \mathbb{R}$ where $J$ is an open subset of $\mathbb{R}$ (the domain of definition of $\mathcal{H}_{0}$ ), and consequently $\mathcal{H}_{0}\left(I_{1}\right) \in \mathbb{R}$. Therefore $\mathcal{H}_{0}^{\prime}$ means derivative with respect to the variable $I_{1}$.

The differential system (2) is defined on the energy level $\mathcal{H}\left(I_{1}, \ldots, I_{n}, \theta_{1}, \ldots, \theta_{n}\right)$ $=h^{*}$ with $h^{*} \in \mathbb{R}$, and we assume that the value $h^{*}$ is such that the function $\mathcal{H}_{0}^{-1}$ in a neighborhood of $h^{*}$ is a diffeomorphism. Therefore the expression $\mathcal{H}_{0}^{\prime}\left(\mathcal{H}_{0}^{-1}\left(h^{*}\right)\right)$ is well defined.

On the other hand, every periodic solution of a differential system has defined in its neighborhood a return map $F$ usually called the Poincaré map. The periodic solution provides a fixed point of the map $F$. The stability or instability of this fixed point for the map $F$ is what we call the stability behavior of the Poincaré map associated to the periodic solution in the statement of Theorem 1. For more details on the Poincaré map see for instance [15].

Theorem 1 will be proved in section 2 .

The next objective of the present work is to study the periodic orbits of the Hamiltonian system with the perturbed Keplerian Hamiltonian of the form

$$
\mathcal{H}=\frac{1}{2}\left(P_{1}^{2}+P_{2}^{2}+P_{3}^{2}\right)-\frac{1}{\sqrt{Q_{1}^{2}+Q_{2}^{2}+Q_{3}^{2}}}+\varepsilon \mathcal{P}_{1}\left(Q_{1}^{2}+Q_{2}^{2}, Q_{3}\right) .
$$

Note that the perturbation is symmetric with respect to the $Q_{3}$-axis. It is easy to check that the third component $K=Q_{1} P_{2}-Q_{2} P_{1}$ of the angular momentum is a first integral of the Hamiltonian system associated to the Hamiltonian (3). We use this second first integral to simplify the analysis of the given axially symmetric Keplerian perturbed system.

In the following we use the Delaunay variables for studying easily the periodic orbits of the Hamiltonian system associated to the Hamiltonian (3), see 
$[4,13]$ for more details on the Delaunay variables. Thus, in Delaunay variables the Hamiltonian (3) has the form

$$
\mathcal{H}=-\frac{1}{2 L^{2}}+\varepsilon \mathcal{P}(l, g, k, L, G, K)=-\frac{1}{2 L^{2}}+\varepsilon \mathcal{P}(l, g, L, G, K),
$$

where $l$ is the mean anomaly, $g$ is the argument of the perigee of the unperturbed elliptic orbit measured in the invariant plane, $k$ is the longitude of the node, $L$ is the square root of the semi-major axis of the unperturbed elliptic orbit, $G$ is the modulus of the total angular momentum and $K$ is the third component of the angular momentum. Moreover, $\mathcal{P}$ is the perturbation obtained from the perturbation $\mathcal{P}_{1}$ using the transformation to Delaunay variables, namely

$$
\begin{aligned}
& Q_{1}=r(\cos (f+g) \cos k-c \sin (f+g) \sin k), \\
& Q_{2}=r(\cos (f+g) \sin k+c \sin (f+g) \cos k), \\
& Q_{3}=r s \sin (f+g),
\end{aligned}
$$

with

$$
c=\frac{K}{G}, \quad s^{2}=1-\frac{K^{2}}{G^{2}} .
$$

The true anomaly $f$ and the eccentric anomaly $E$ are auxiliary quantities defined by the relations

$$
\begin{gathered}
\sqrt{1-e^{2}}=\frac{G}{L}, \quad r=a(1-e \cos E), \quad l=E-e \sin E . \\
\sin f=\frac{a \sqrt{1-e^{2}} \sin E}{r}, \quad \cos f=\frac{a(\cos E-e)}{r},
\end{gathered}
$$

where $e$ is the eccentricity of the unperturbed elliptic orbit.

Note that the angular variable $k$ is a cyclic variable for the Hamiltonian (4), and consenquently $K$ is a first integral of the Hamiltonian system as we already knew.

The family of Hamiltonians (4) is a particular subclass of the Hamiltonians (1) with $\mathcal{H}_{1}=\mathcal{P}$. We denote by $\langle\mathcal{P}\rangle$ the averaged map of $\mathcal{P}$ with respect to the mean anomaly $l$, i.e.,

$$
\langle\mathcal{P}\rangle=\frac{1}{2 \pi} \int_{0}^{2 \pi} \mathcal{P}(l, g, L, G, K) d l=\frac{1}{2 \pi} \int_{0}^{2 \pi} \mathcal{P}(E-e \sin E, g, L, G, K)(1-e \cos E) d E .
$$

We remark that the map $\langle\mathcal{P}\rangle$ only depends on the angle $g$ and the three action variables $L, G, K$. We claim that $\mathcal{H}_{0}^{\prime}\left(\mathcal{H}_{0}^{-1}\left(h^{*}\right)\right)=\left(-2 h^{*}\right)^{3 / 2}$. Indeed $\mathcal{H}_{0}(L)=-1 /\left(2 L^{2}\right)=h^{*}$, so $\mathcal{H}_{0}^{-1}\left(h^{*}\right)=\left(-2 h^{*}\right)^{1 / 2}$. Since $\mathcal{H}_{0}^{\prime}(L)=1 / L^{3}$, the 
claim follows. We also have from the definition of Poisson parenthesis that

$$
\begin{aligned}
& \{G,\langle\mathcal{P}\rangle\}=-\frac{\partial G}{\partial G} \frac{\partial\langle\mathcal{P}\rangle}{\partial g}=-\frac{\partial\langle\mathcal{P}\rangle}{\partial g}, \\
& \{g,\langle\mathcal{P}\rangle\}=\frac{\partial g}{\partial g} \frac{\partial\langle\mathcal{P}\rangle}{\partial G}=\frac{\partial\langle\mathcal{P}\rangle}{\partial G}, \\
& \{k,\langle\mathcal{P}\rangle\}=\frac{\partial k}{\partial k} \frac{\partial\langle\mathcal{P}\rangle}{\partial K}=\frac{\partial\langle\mathcal{P}\rangle}{\partial K} .
\end{aligned}
$$

Then, by Theorem 1 at the energy level $\mathcal{H}=h^{*}$ with $h^{*}<0$ (because $\mathcal{H}_{0}(L)=$ $\left.-1 /\left(2 L^{2}\right)\right)$ and with angular momentum $K=k^{*}$, the differential system $(2)$ with respect to the mean anomaly $l$ is

$$
\begin{aligned}
\frac{d G}{d l} & =\varepsilon \frac{\{G,\langle\mathcal{P}\rangle\}}{\mathcal{H}_{0}^{\prime}\left(\mathcal{H}_{0}^{-1}\left(h^{*}\right)\right)}=-\varepsilon\left(-2 h^{*}\right)^{3 / 2} \frac{\partial\langle\mathcal{P}\rangle}{\partial g}=-\varepsilon f_{1}(g, G, K), \\
\frac{d g}{d l} & =\varepsilon \frac{\{g,\langle\mathcal{P}\rangle\}}{\mathcal{H}_{0}^{\prime}\left(\mathcal{H}_{0}^{-1}\left(h^{*}\right)\right)}=\varepsilon\left(-2 h^{*}\right)^{3 / 2} \frac{\partial\langle\mathcal{P}\rangle}{\partial G}=\varepsilon f_{2}(g, G, K), \\
\frac{d k}{d l} & =\varepsilon \frac{\{k,\langle\mathcal{P}\rangle\}}{\mathcal{H}_{0}^{\prime}\left(\mathcal{H}_{0}^{-1}\left(h^{*}\right)\right)}=\varepsilon\left(-2 h^{*}\right)^{3 / 2} \frac{\partial\langle\mathcal{P}\rangle}{\partial K}=\varepsilon f_{3}(g, G, K) .
\end{aligned}
$$

Note that we do not write the differential equation $d K / d t=0$ because we are working in the invariant set $\mathcal{H}=h^{*}$ and $K=k^{*}$.

Now we are ready to state a corollary of Theorem 1 which provides sufficient conditions for the existence and the kind of stability of the periodic orbits in the perturbed Kepler problems with axial symmetry.

Corollary 2. System (6) is the Hamiltonian system taking as independent variable the mean anomaly $l$ of the Hamiltonian (3) written in Delaunay variables on the fixed energy level $\mathcal{H}=h^{*}<0$ and on the fixed third component of the angular momentum $K=k^{*}$. If $\varepsilon \neq 0$ is sufficiently small then for every solution $p=\left(g_{0}, G_{0}, k^{*}\right)$ of the system $f_{i}(g, G, K)=0$ for $i=1,2,3$ satisfying that

$$
\operatorname{det}\left(\left.\frac{\partial\left(f_{1}, f_{2}, f_{3}\right)}{\partial(g, G, K)}\right|_{(g, G, K)=\left(g_{0}, G_{0}, k^{*}\right)}\right) \neq 0,
$$

and all $k_{0} \in[0,2 \pi)$ there exists a $2 \pi$-periodic solution $\gamma_{\varepsilon}(l)=(g(l, \varepsilon), k(l, \varepsilon)$, $\left.L(l, \varepsilon), G(l, \varepsilon), K(l, \varepsilon)=k^{*}\right)$ such that $\gamma_{\varepsilon}(0) \rightarrow\left(g_{0}, k_{0}, \sqrt{-2 h^{*}}, G_{0}, k^{*}\right)$ when $\varepsilon \rightarrow 0$. The stability or instability of the periodic solution $\gamma_{\varepsilon}(l)$ is given by the stability or instability of the equilibrium point $p$ of system (6). In fact, the equilibrium point $p$ has the stability behavior of the Poincare map associated to the periodic solution $\gamma_{\varepsilon}(l)$.

We remark that the fact that we have a periodic solution for every $k_{0} \in$ $[0,2 \pi)$ with the same initial conditions for all the other variables, means that we really have a 2 -dimensional torus foliated by periodic solutions.

There are many articles studying the periodic orbits of different perturbed Keplerian problems, see for instance $[8,9,16]$ and the papers quoted therein. 
In what follows we shall study three Hamiltonian systems with Hamiltonian of the form (4). The first one will be the Matese-Whitman Hamiltonian system modeling the galactic tidal interaction with the Oort comet cloud. The second one is the spatial anisotropic Kepler problem which analyzes the isotropy of the space. Finally, we consider the spatial generalized van der Waals Hamiltonian system modeling the dynamical symmetries of the perturbed hydrogen atom.

The Hamiltonian system associated to the Hamiltonian

$$
\mathcal{H}=\frac{1}{2}\left(P_{1}^{2}+P_{2}^{2}+P_{3}^{2}\right)-\frac{1}{\sqrt{Q_{1}^{2}+Q_{2}^{2}+Q_{3}^{2}}}+\varepsilon Q_{3}^{2}
$$

was proposed in the seminal papers of Matese and Whitman [11, 12] for studying the dynamics of the Oort cloud [14], see also [10]. In fact it is a simple dynamical system which consider the heliocentric Kepler problem perturbed by the quadratic tidal potential of the Galaxy. Note that the Hamiltonian (8) is a particular case of the Hamiltonian (3).

We have the following result on the periodic orbits and their kind of stability for the Hamiltonian system associated to the Hamiltonian (8).

Theorem 3. On every energy level $\mathcal{H}=h^{*}<0$ and for the third component of the angular momentum $K=k^{*}=0$, the Matese-Whitman Hamiltonian system associated to the Hamiltonian (8) for $\varepsilon \neq 0$ sufficiently small has two $2 \pi$-periodic solution $\left.\gamma_{\varepsilon}^{ \pm}(l)=(g(l, \varepsilon), k(l, \varepsilon)), L(l, \varepsilon), G(l, \varepsilon), K(l, \varepsilon)\right)$ such that

$$
\gamma_{\varepsilon}^{ \pm}(0) \rightarrow\left( \pm \frac{1}{2} \arccos \left(\frac{3}{5}\right), k_{0}, \frac{1}{\sqrt{-2 h^{*}}}, \frac{1}{\sqrt{-2 h^{*}}}, 0\right) \text { when } \varepsilon \rightarrow 0,
$$

for each $k_{0} \in[0,2 \pi)$. These periodic orbits have a stable manifold of dimension 2 and an unstable of dimension 1 , and consequently are unstable.

As we have mention after the statement of Corollary 2 the fact that we have a periodic orbit for each $k_{0} \in[0,2 \pi)$ means that we have two 2 -dimensional torus foliated by periodic solutions for each value of $g_{0}= \pm \frac{1}{2} \arccos \left(\frac{3}{5}\right)$. Putting together the invariant manifolds of each of these periodic orbits, we obtain that these 2-dimensional tori have a stable manifold of dimension 3 and an unstable of dimension 2 .

Theorem 3 is proved in section 3 .

We note that the values $\pm 26.6 \ldots= \pm \arccos (3 / 5) / 2$ also appear in the paper [11], being there a boundary for the argument of the perigee of the periodic orbits of the Hamiltonian system with Hamiltonian (8).

Now we shall analyze the so called anisotropic spatial Kepler problem given by the Hamiltonian

$$
\mathcal{H}=\frac{1}{2}\left(P_{1}^{2}+P_{2}^{2}+P_{3}^{2}\right)-\frac{1}{\sqrt{\mu\left(Q_{1}^{2}+Q_{2}^{2}\right)+Q_{3}^{2}}}
$$

where $\mu$ is a positive real parameter. This problem originally comes from the quantum mechanic, see for instance $[7,2,3]$. 
We want to study its periodic orbits for values of the parameter $\mu$ close to 1. Therefore define $\varepsilon=1-\mu$, expanding in Taylor series in $\varepsilon$ we obtain $\mathcal{H}=\frac{1}{2}\left(P_{1}^{2}+P_{2}^{2}+P_{3}^{2}\right)-\frac{1}{\sqrt{Q_{1}^{2}+Q_{2}^{2}+Q_{3}^{2}}}-\varepsilon \frac{Q_{1}^{2}+Q_{2}^{2}}{2\left(Q_{1}^{2}+Q_{2}^{2}+Q_{3}^{2}\right)^{3 / 2}}+O\left(\varepsilon^{2}\right)$.

Note that this Hamiltonian is of the form (3).

Theorem 4. On every energy level $\mathcal{H}=h^{*}<0$ and for the third component of the angular momentum $K=k^{*}=0$, the spatial anisotropic Kepler Hamiltonian system associated to the Hamiltonian (9) for $\varepsilon \neq 0$ sufficiently small has two $2 \pi$-periodic solution $\left.\gamma_{\varepsilon}^{ \pm}(l)=(g(l, \varepsilon), k(l, \varepsilon)), L(l, \varepsilon), G(l, \varepsilon), K(l, \varepsilon)\right)$ such that

$$
\gamma_{\varepsilon}^{ \pm}(0) \rightarrow\left( \pm \frac{\pi}{4}, k_{0}, \frac{1}{\sqrt{-2 h^{*}}}, \frac{1}{\sqrt{-2 h^{*}}}, 0\right) \text { when } \varepsilon \rightarrow 0,
$$

for each $k_{0} \in[0,2 \pi)$. These periodic orbits have a stable manifold of dimension 2 and an unstable of dimension 1 , and consequently are unstable.

Theorem 4 is proved in section 4 .

The generalized van der Waals Hamiltonian system was proposed in the paper [1] via the following Hamiltonian with $\beta \in \mathbb{R}$

$$
\mathcal{H}=\frac{1}{2}\left(P_{1}^{2}+P_{2}^{2}+P_{3}^{2}\right)-\frac{1}{\sqrt{Q_{1}^{2}+Q_{2}^{2}+Q_{3}^{2}}}+\varepsilon\left(Q_{1}^{2}+Q_{2}^{2}+\beta^{2} Q_{3}^{2}\right) .
$$

Note that this Hamiltonian is of the form (3). For more references on this Hamiltonian system see the ones quoted in [6].

Theorem 5. On every energy level $\mathcal{H}=h^{*}<0$ and for the third component of the angular momentum $K=k^{*}$, the spatial van der Waals Hamiltonian system associated to the Hamiltonian (10) for $\varepsilon \neq 0$ sufficiently small has:

(a) For $K=k^{*}=0$ two $2 \pi$-periodic solution $\gamma_{\varepsilon}^{ \pm}(l)=(g(l, \varepsilon), k(l, \varepsilon)), L(l, \varepsilon)$, $G(l, \varepsilon), K(l, \varepsilon))$ such that

$$
\gamma_{\varepsilon}^{ \pm}(l)(0) \rightarrow\left( \pm \frac{1}{2} \arccos \left(\frac{3\left(\beta^{2}+1\right)}{5\left(\beta^{2}-1\right)}\right), k_{0}, \frac{1}{\sqrt{-2 h^{*}}}, \frac{1}{\sqrt{-2 h^{*}}}, 0\right) \text { when } \varepsilon \rightarrow 0
$$

for each $k_{0} \in[0,2 \pi)$ if $\beta \in(-\infty,-2) \cup(-1 / 2,1 / 2) \cup(2, \infty)$. These periodic orbits have a stable manifold of dimension 2 and an unstable of dimension 1 if $\beta \in(-1 / 2,1 / 2)$, and have a stable manifold of dimension 1 and an unstable of dimension 2 if $\beta \in(-\infty,-2) \cup(2, \infty)$. Consequently these periodic orbits are unstable.

(b) For $K=k^{*} \neq 0$ four $2 \pi$-periodic solutions $\gamma_{\varepsilon}^{ \pm, \pm}(l)=(g(l, \varepsilon), k(l, \varepsilon))$, $L(l, \varepsilon), G(l, \varepsilon), K(l, \varepsilon))$ such that

$$
\gamma_{\varepsilon}^{ \pm, \pm}(0) \rightarrow\left( \pm \frac{\pi}{2}, k_{0}, \frac{1}{\sqrt{-2 h^{*}}}, \frac{1}{2} \sqrt{\frac{5}{-2 h^{*}}}, \pm \frac{1}{4} \sqrt{\frac{5\left(1-4 \beta^{2}\right)}{-2 h^{*}\left(1-\beta^{2}\right)}}\right) \text { when } \varepsilon \rightarrow 0 \text {, }
$$

for each $k_{0} \in[0,2 \pi)$ if $\beta \in(-1,-1 / 2) \cup(1 / 2,1)$. 
Theorem 5 is proved in section 5 .

The result of statement (a) of Theorem 5 was already obtained using cylindrical coordinates in [6].

The stability or instability of the four periodic orbits of statement (b) of Theorem 5 can be determined analyzing the eigenvalues of the corresponding Jacobian matrices, but since the expression of these eigenvalues are huge and depend on the two parameters $h^{*}$ and $\beta$, this study is a long task that we do not do here.

We remark that when $\left(\beta^{2}-1\right)\left(\beta^{2}-4\right)\left(\beta^{2}-1 / 4\right)=0$, i.e. for the values that the averaging theory for finding periodic orbits do not provide any information, it is known that for those values of $\beta$ the van der Waals Hamiltonian system is integrable, see [5]. Therefore, the averaging method when cannot be applied for finding periodic orbits provides a suspicion that for such values of the parameter the system could be integrable.

Remark 6. The periodic orbits which persist for $\varepsilon \neq 0$ sufficiently small in Theorems 3, 4 and 5(a) bifurcate from elliptic periodic orbits of the unperturbed Kepler problem contained in the plane defined by the orbit of the two bodies of the Kepler problem. The periodic orbits of Theorem 5(b) bifurcate from elliptic periodic orbits of the unperturbed Kepler problem not contained in the plane defined by the orbit of the two bodies of the Kepler problem

Remark 6 is proved inside the proofs of Theorems 3,4 and 5 .

\section{Averaged Hamiltonian in aCtion-Angle variables}

The Hamiltonian system associated to the Hamiltonian (1) can be written as

$$
\begin{aligned}
\frac{d I_{i}}{d t} & =\varepsilon\left\{I_{i}, \mathcal{H}_{1}\right\}=-\varepsilon \frac{\partial \mathcal{H}_{1}}{\partial \theta_{i}} \quad i=1, \ldots, n, \\
\frac{d \theta_{i}}{d t} & =\varepsilon\left\{\theta_{i}, \mathcal{H}_{1}\right\}=\varepsilon \frac{\partial \mathcal{H}_{1}}{\partial I_{i}} \quad i=2, \ldots, n, \\
\frac{d \theta_{1}}{d t} & =\mathcal{H}_{0}^{\prime}\left(I_{1}\right)+\varepsilon\left\{\theta_{1}, \mathcal{H}_{1}\right\}=\mathcal{H}_{0}^{\prime}\left(I_{1}\right)+\varepsilon \frac{\partial \mathcal{H}_{1}}{\partial I_{1}} .
\end{aligned}
$$

Lemma 7. Taking as new independent variable the variable $\theta_{1}$ we have in the fixed energy level $\mathcal{H}=h^{*}<0$ that the differential system (11) becomes

$$
\begin{aligned}
\frac{d I_{i}}{d \theta_{1}} & =\varepsilon \frac{\left\{I_{i}, \mathcal{H}_{1}\right\}}{\mathcal{H}_{0}^{\prime}\left(\mathcal{H}_{0}^{-1}\left(h^{*}\right)\right)}+O\left(\varepsilon^{2}\right), i=2, \ldots, n \\
\frac{d \theta_{i}}{d \theta_{1}} & =\varepsilon \frac{\left\{\theta_{i}, \mathcal{H}_{1}\right\}}{\mathcal{H}_{0}^{\prime}\left(\mathcal{H}_{0}^{-1}\left(h^{*}\right)\right)}+O\left(\varepsilon^{2}\right), i=2, \ldots, n
\end{aligned}
$$

with $I_{1}=\mathcal{H}_{0}^{-1}\left(h^{*}\right)+O(\varepsilon)$ if $\mathcal{H}_{0}^{\prime}\left(\mathcal{H}_{0}^{-1}\left(h^{*}\right)\right) \neq 0$. 
Proof. Taking as new independent variable $\theta_{1}$, the equations (11) become

$$
\begin{aligned}
& \frac{d I_{i}}{d \theta_{1}}=\frac{\varepsilon\left\{I_{i}, \mathcal{H}_{1}\right\}}{\mathcal{H}_{0}^{\prime}\left(I_{1}\right)+\varepsilon\left\{\theta_{1}, \mathcal{H}_{1}\right\}}=\varepsilon \frac{\left\{I_{i}, \mathcal{H}_{1}\right\}}{\mathcal{H}_{0}^{\prime}\left(I_{1}\right)}+O\left(\varepsilon^{2}\right) \quad i=1, \ldots, n, \\
& \frac{d \theta_{i}}{d \theta_{1}}=\frac{\varepsilon\left\{\theta_{i}, \mathcal{H}_{1}\right\}}{\mathcal{H}_{0}^{\prime}\left(I_{1}\right)+\varepsilon\left\{\theta_{1}, \mathcal{H}_{1}\right\}}=\varepsilon \frac{\left\{\theta_{i}, \mathcal{H}_{1}\right\}}{\mathcal{H}_{0}^{\prime}\left(I_{1}\right)}+O\left(\varepsilon^{2}\right) \quad i=2, \ldots, n .
\end{aligned}
$$

Fixing the energy level of $\mathcal{H}=h^{*}<0$ we obtain $h^{*}=\mathcal{H}_{0}\left(I_{1}\right)+\varepsilon \mathcal{H}_{1}\left(I_{1}, \ldots, I_{n}, \theta_{1}\right.$, $\left.\ldots, \theta_{n}\right)$. Using the Implicit Function Theorem and the fact that $\mathcal{H}_{0}^{\prime}\left(\mathcal{H}_{0}^{-1}\left(h^{*}\right)\right) \neq$ 0 , for $\varepsilon$ sufficiently small, we get $I_{1}=\mathcal{H}_{0}^{-1}\left(h^{*}\right)+O(\varepsilon)$, and the equations are reduced to (12).

Proof of Theorem 1. The averaged system in the angle $\theta_{1}$ obtained from (12) is

$$
\begin{gathered}
\frac{d I_{i}}{d \theta_{1}}=-\frac{1}{2 \pi} \frac{\varepsilon}{\mathcal{H}_{0}^{\prime}\left(\mathcal{H}_{0}^{-1}(h)\right)} \int_{0}^{2 \pi} \frac{\partial \mathcal{H}_{1}}{\partial \theta_{i}} d \theta_{1} \quad i=2, \ldots, n, \\
\frac{d \theta_{i}}{d \theta_{1}}=\frac{1}{2 \pi} \varepsilon \frac{\left\{\theta_{i}, \mathcal{H}_{1}\right\}}{\mathcal{H}_{0}^{\prime}\left(\mathcal{H}_{0}^{-1}(h)\right)} \int_{0}^{2 \pi} \frac{\partial \mathcal{H}_{1}}{\partial I_{i}} d \theta_{1} \quad i=2, \ldots, n .
\end{gathered}
$$

See the Appendix for a short introduction to the averaging theory used in this paper.

Since,

$$
\begin{gathered}
\frac{\partial\left\langle\mathcal{H}_{1}\right\rangle}{\partial \theta_{i}}=\frac{1}{2 \pi} \int_{0}^{2 \pi} \frac{\partial \mathcal{H}_{1}}{\partial \theta_{i}} d \theta_{1} \quad i=2, \ldots, n, \\
\frac{\partial\left\langle\mathcal{H}_{1}\right\rangle}{\partial I_{i}}=\frac{1}{2 \pi} \int_{0}^{2 \pi} \frac{\partial \mathcal{H}_{1}}{\partial I_{i}} d \theta_{1} \quad i=2, \ldots, n,
\end{gathered}
$$

the differential system (13) becomes

$$
\begin{aligned}
\frac{d I_{i}}{d \theta_{1}} & =-\frac{\varepsilon}{\mathcal{H}_{0}^{\prime}\left(\mathcal{H}_{0}^{-1}(h)\right)} \frac{\partial\left\langle\mathcal{H}_{1}\right\rangle}{\partial \theta_{i}}=\varepsilon \frac{\left\{I_{i},\left\langle\mathcal{H}_{1}\right\rangle\right\}}{\mathcal{H}_{0}^{\prime}\left(\mathcal{H}_{0}^{-1}(h)\right)} \quad i=2, \ldots, n, \\
\frac{d \theta_{i}}{d \theta_{1}} & =\frac{\varepsilon}{\mathcal{H}_{0}^{\prime}\left(\mathcal{H}_{0}^{-1}(h)\right)} \frac{\partial\left\langle\mathcal{H}_{1}\right\rangle}{\partial I_{i}}=\varepsilon \frac{\left\{\theta_{i},\left\langle\mathcal{H}_{1}\right\rangle\right\}}{\mathcal{H}_{0}^{\prime}\left(\mathcal{H}_{0}^{-1}(h)\right)} \quad i=2, \ldots, n,
\end{aligned}
$$

which coincides with the system (2).

Once we have obtained the averaged system (2) it is immediate to check that it satisfies the assumptions of Theorem 8 of the Appendix, then applying the conclusions of Theorem 8 to our averaged system (2) the rest of the statement of Theorem 1 follows immediately. 


\section{The Matese-Whitman problem}

For the Matese-Whitman Hamiltonian the function $\mathcal{P}(E, g, L, G, K)$ given in (4) is

$$
\begin{aligned}
& \frac{\left(G^{2}-K^{2}\right)(e \cos E-1)^{2} L^{4}}{2 G^{2}}-\frac{L^{4}\left(G^{2}-K^{2}\right)(e-\cos E)^{2} \cos ^{2} g}{2 G^{2}} \\
& +\frac{L^{4}\left(G^{2}-K^{2}\right)(e-\cos E)^{2} \sin ^{2} g}{2 G^{2}} \\
& -\frac{2 L^{3}\left(G^{2}-K^{2}\right)(e-\cos E) \cos g \sin E \sin g}{G} \\
& +\frac{1}{2} L^{2}\left(G^{2}-K^{2}\right) \cos ^{2} g \sin ^{2} E-\frac{1}{2} L^{2}\left(G^{2}-K^{2}\right) \sin ^{2} E \sin ^{2} g .
\end{aligned}
$$

Its averaged function $\langle\mathcal{P}\rangle$ with respect the mean anomaly $l$ is

$$
\begin{aligned}
\langle\mathcal{P}\rangle & =\frac{1}{2 \pi} \int_{0}^{2 \pi} \mathcal{P}(E, g, L, G, K)(1-e \cos E) d E \\
& =\frac{L^{2}\left(G^{2}-K^{2}\right)\left(5 L^{2}-3 G^{2}+5\left(G^{2}-L^{2}\right) \cos (2 g)\right)}{4 G^{2}} .
\end{aligned}
$$

The equations (6) for our system are the averaged equations of the Hamiltonian system with Hamiltonian (8)

$$
\begin{aligned}
& \frac{d G}{d l}=\varepsilon \frac{5\left(1+2 h^{*} G^{2}\right)\left(G^{2}-K^{2}\right) \sin (2 g)}{2 \sqrt{-2 h^{*}} G^{2}}=-\varepsilon f_{1}(g, G, K), \\
& \frac{d g}{d l}=-\varepsilon \frac{6 G^{4} h^{*}+5 K^{2}-5\left(2 G^{4} h^{*}+K^{2}\right) \cos (2 g)}{2 \sqrt{-2 h^{*}} G^{3}}=\varepsilon f_{2}(g, G, K), \\
& \frac{d k}{d l}=\varepsilon \frac{K\left(5\left(1+2 h^{*} G^{2}\right) \cos (2 g)-5-6 h^{*} G^{2}\right)}{2 \sqrt{-2 h^{*}} G^{2}}=\varepsilon f_{3}(g, G, K),
\end{aligned}
$$

here $L=1 / \sqrt{-2 h^{*}}+O(\varepsilon)$, see for more details the proof of Theorem 1 . The equilibrium solutions $\left(g_{0}, G_{0}, k^{*}\right)$ of this averaged system satisfying (7) give rise to periodic orbits of the Hamiltonian system with Hamiltonian (8) for each $\mathcal{H}=h^{*}<0$ and $K=k^{*}$, see Theorem 8. There are only two of such equilibria, namely

$$
\left(g_{0}, G_{0}, k^{*}\right)=\left( \pm \frac{1}{2} \arccos \left(\frac{3}{5}\right), \frac{1}{\sqrt{-2 h^{*}}}, 0\right) .
$$

The Jacobian (7) at these equilibra is equal to $16 \sqrt{-2 h^{*}} \neq 0$. So each one of these equilibria provides for each value of $k \in[0,2 \pi)$ one periodic orbit of the Hamiltonian system with Hamiltonian (8) for each $\mathcal{H}=h^{*}<0$ and $K=k^{*}=0$.

Since the third component of the angular momentum $K$ of these periodic orbits is zero, these periodic orbits bifurcate from elliptic periodic orbits (note that the argument of the perigee $g_{0} \neq 0$ ) of the Kepler problem contained in 
the plane of motion of the two bodies defining the Kepler problem. Moreover, since the eigenvalues of the matrix

$$
\left(\left.\frac{\partial\left(f_{1}, f_{2}, f_{3}\right)}{\partial(g, G, K)}\right|_{(g, G, K)=\left(g_{0}, G_{0}, k^{*}\right)}\right)
$$

at these equilibria are \pm 4 and $-\sqrt{-2 h^{*}}$, these periodic orbits have a stable manifold of dimension 2 and an unstable of dimension 1 . This completes the proof of Theorem 3.

\section{The Anisotropic Kepler problem}

For the anisotropic Kepler problem the function $\mathcal{P}(E, g, h, G, K)$ is equal to

$$
\begin{aligned}
& -\frac{\left(G^{2}-K^{2}\right) \sin ^{2} E \cos ^{2} g}{4 L^{4}(e \cos E-1)^{3}}+\frac{\left(G^{2}-K^{2}\right)(e-\cos E)^{2} \cos ^{2} g}{4 G^{2} L^{2}(e \cos E-1)^{3}} \\
& +\frac{\left(G^{2}-K^{2}\right)(e-\cos E) \sin E \sin g \cos g}{G L^{3}(e \cos E-1)^{3}}+\frac{\left(G^{2}-K^{2}\right) \sin ^{2} E \sin ^{2} g}{4 L^{4}(e \cos E-1)^{3}} \\
& -\frac{\left(G^{2}-K^{2}\right)(e-\cos E)^{2} \sin ^{2} g}{4 G^{2} L^{2}(e \cos E-1)^{3}}+\frac{G^{2}+K^{2}}{4 G^{2} L^{2}(e \cos E-1)}
\end{aligned}
$$

Its averaged function with respect to the mean anomaly is

$$
\begin{aligned}
\langle\mathcal{P}\rangle & =\frac{1}{2 \pi} \int_{0}^{2 \pi} \mathcal{P}(E, g, h, G, K)(1-e \cos E) d E \\
& =-\frac{\left(G^{2}+K^{2}\right)(G+L)-\left(G^{2}-K^{2}\right)(G-L) \cos (2 g)}{4 G^{2} L^{2}(G+L)} .
\end{aligned}
$$

The equations (6) are the averaged equations of the Hamiltonian system with Hamiltonian (9)

$$
\begin{aligned}
\frac{d G}{d l} & =\varepsilon \frac{4\left(1-G \sqrt{-2 h^{*}}\right)\left(-h^{*}\right)^{5 / 2}\left(G^{2}-K^{2}\right) \sin (2 g)}{\sqrt{2} G^{2}\left(1+G \sqrt{-2 h^{*}}\right)}=-\varepsilon f_{1}(g, G, K), \\
\frac{d g}{d l} & =\varepsilon \frac{A}{G^{3}\left(1+G \sqrt{-2 h^{*}}\right)^{2}}=\varepsilon f_{2}(g, G, K), \\
\frac{d k}{d l} & =\varepsilon \frac{2\left(h^{*}\right)^{2} K\left(2 h^{*} G-\sqrt{-2 h^{*}}+\left(\sqrt{-2 h^{*}}+2 h^{*} G\right) \cos (2 g)\right)}{G^{2}\left(1+G \sqrt{-2 h^{*}}\right)}=\varepsilon f_{3}(g, G, K),
\end{aligned}
$$

where

$$
\begin{aligned}
A= & 4\left(-h^{*}\right)^{5 / 2}\left(\left(-1-2 G \sqrt{-2 h^{*}}+2 G^{2} h^{*}\right) K^{2}\right)+ \\
& \left.\left(-G^{3} \sqrt{-2 h^{*}}+K^{2}+G \sqrt{-2 h^{*}} K^{2}+2 h^{*} G^{2} K^{2}\right) \cos (2 g)\right),
\end{aligned}
$$

here $L=1 / \sqrt{-2 h^{*}}+O(\varepsilon)$. The equilibrium solutions $\left(g_{0}, G_{0}, k^{*}\right)$ of this averaged system satisfying (7) give rise to periodic orbits of the Hamiltonian 
system with Hamiltonian (9) for each $\mathcal{H}=h^{*}<0$ and $K=k^{*}$, see Theorem 8. There are two of such equilibria, namely

$$
\left(g_{0}, G_{0}, k^{*}\right)=\left( \pm \frac{\pi}{4}, \frac{1}{\sqrt{-2 h^{*}}}, 0\right) .
$$

The Jacobian $(7)$ at these equilibra is equal to $16 \sqrt{2}\left(-h^{*}\right)^{19 / 2} \neq 0$. So each of these equilibria provide one periodic orbit for each value of $k \in[0,2 \pi)$ of the Hamiltonian system with Hamiltonian (9) for each $\mathcal{H}=h^{*}<0$ and $K=k^{*}=$ 0 . Since the third component of the angular momentum is zero, these periodic orbits bifurcate from elliptic orbits $\left(g_{0} \neq 0\right)$ of the Kepler problem contained in the plane of motion of the two bodies. Moreover, since the eigenvalues of the matrix (14) at these equilibra are $\pm 2\left(h^{*}\right)^{3}$ and $-4 \sqrt{2}\left(-h^{*}\right)^{7 / 2}$, these periodic orbits have a stable manifold of dimension 2 and an unstable of dimension 1. This completes the proof of Theorem 4 .

\section{The Generalized VAN DER WAals Hamiltonian}

For the generalized van der Waals Hamiltonian system the function $\mathcal{P}(E, g, h, G, K)$ is equal to

$$
\begin{aligned}
& \frac{\left(\beta^{2} G^{2}+G^{2}+K^{2}-K^{2} \beta^{2}\right)(e \cos E-1)^{2} L^{4}}{2 G^{2}}- \\
& \frac{L^{4}\left(G^{2}-K^{2}\right)\left(\beta^{2}-1\right)(e-\cos E)^{2} \cos ^{2} g}{2 G^{2}}+ \\
& \frac{L^{4}\left(G^{2}-K^{2}\right)\left(\beta^{2}-1\right)(e-\cos E)^{2} \sin ^{2} g}{2 G^{2}} \\
& -\frac{2 L^{3}\left(G^{2}-K^{2}\right)\left(\beta^{2}-1\right)(e-\cos E) \cos g \sin E \sin g}{G} \\
& +\frac{1}{2} L^{2}\left(G^{2}-K^{2}\right)\left(\beta^{2}-1\right) \cos ^{2} g \sin ^{2} E \\
& -\frac{1}{2} L^{2}\left(G^{2}-K^{2}\right)\left(\beta^{2}-1\right) \sin ^{2} E \sin ^{2} g
\end{aligned}
$$

Its averaged function with respect to the mean anomaly is

$$
\langle\mathcal{P}\rangle=\frac{1}{2 \pi} \int_{0}^{2 \pi} \mathcal{P}(E, g, h, G, K)(1-e \cos E) d E=\frac{B}{4 G^{2}},
$$

where $B=L^{2}\left(5\left(G^{2}-K^{2}\right)\left(G^{2}-L^{2}\right)\left(\beta^{2}-1\right) \cos (2 g)-\left(3 G^{2}-5 L^{2}\right)\left(G^{2}+K^{2}+\right.\right.$ $\left.\left.\left(G^{2}-K^{2}\right) \beta^{2}\right)\right)$. 
The equations (6) are the averaged equations of the Hamiltonian system with Hamiltonian (10)

$$
\begin{aligned}
\frac{d G}{d l} & =\varepsilon \frac{5\left(1+2 h^{*} G^{2}\right)\left(G^{2}-K^{2}\right)\left(\beta^{2}-1\right) \sin (2 g)}{2 G^{2} \sqrt{-2 h^{*}}}=-\varepsilon f_{1}(g, G, K), \\
\frac{d g}{d l} & =-\varepsilon \frac{C}{2 G^{3} \sqrt{-2 h^{*}}}=\varepsilon f_{2}(g, G, K), \\
\frac{d k}{d l} & =\varepsilon \frac{K\left(\beta^{2}-1\right)\left(-5-6 h^{*} G^{2}+5\left(1+2 h^{*} G^{2}\right) \cos (2 g)\right)}{2 G^{2} \sqrt{-2 h^{*}}}=\varepsilon f_{3}(g, G, K),
\end{aligned}
$$

where $C=5 K^{2}\left(\beta^{2}-1\right)+6 h^{*} G^{4}\left(\beta^{2}+1\right)-5\left(2 h^{*} G^{4}+K^{2}\right)\left(\beta^{2}-1\right) \cos (2 g)$ here $L=1 / \sqrt{-2 h^{*}}+O(\varepsilon)$. The equilibrium solutions $\left(g_{0}, G_{0}, k^{*}\right)$ of this averaged system satisfying (7) give rise to periodic orbits of the Hamiltonian system with Hamiltonian (10) for each $\mathcal{H}=h^{*}<0$ and $K=k^{*}$, see Theorem 8. These equilibria $\left(g_{0}, G_{0}, k^{*}\right)$ are

$$
\left( \pm \frac{1}{2} \arccos \left(\frac{3\left(\beta^{2}+1\right)}{5\left(\beta^{2}-1\right)}\right), \frac{1}{\sqrt{-2 h^{*}}}, 0\right),\left( \pm \frac{\pi}{2}, \frac{1}{2} \sqrt{\frac{5}{-2 h^{*}}}, \pm \frac{1}{4} \sqrt{\frac{5\left(1-4 \beta^{2}\right)}{-2 h^{*}\left(1-\beta^{2}\right)}}\right)
$$

The first two equilibria exist if $3\left(\beta^{2}+1\right) /\left(5\left(\beta^{2}-1\right)\right) \in[-1,1]$, i.e. if $\beta \in$ $(-\infty,-2] \cup[-1 / 2,1 / 2] \cup[2, \infty)$.

The Jacobian (7) of the first equilibrium is equal to $J=16 \sqrt{-2 h^{*}}\left(\beta^{2}-\right.$ 1) $\left(\beta^{2}-4\right)\left(\beta^{2}-1 / 4\right)$. So each of these equilibria when $\beta \in(-\infty,-2) \cup$ $(-1 / 2,1 / 2) \cup(2, \infty)$ provides one periodic orbit of the Hamiltonian system with Hamiltonian (10) for each $\mathcal{H}=h^{*}<0$ and $K=k^{*}=0$. Since $k^{*}=0$ these periodic orbits bifurcate from an elliptic orbit $\left(g_{0} \neq 0\right)$ of the Kepler problem living in the plane of motion of the two bodies of the Kepler problem. Moreover, since the eigenvalues of the matrix (14) at these equilibra are $\pm 2 \sqrt{\left(\beta^{2}-4\right)\left(4 \beta^{2}-1\right)}$ and $\sqrt{-2 h^{*}}\left(\beta^{2}-1\right)$, these periodic orbits have a stable manifold of dimension 2 and an unstable of dimension 1 if $\beta \in(-1 / 2,1 / 2)$, and have a stable manifold of dimension 1 and an unstable of dimension 2 if $\beta \in(-\infty,-2) \cup(2, \infty)$. This proves statement (a) of the theorem.

The last four equilibria exist if $\beta \in(-1,-1 / 2] \cup[1 / 2,1)$ and have Jacobian equal to $J=-15 \sqrt{-2 h^{*}}\left(\beta^{2}-1\right)\left(4 \beta^{2}-1\right)$. So, for each value of $k \in[0,2 \pi)$ these four equilibria when $\beta \in(-1,-1 / 2) \cup(1 / 2,1)$ provide four periodic orbits of the Hamiltonian system with Hamiltonian (10) for each $\mathcal{H}=h^{*}<0$ and $K=k^{*}= \pm \frac{1}{4} \sqrt{\frac{5\left(1-4 \beta^{2}\right)}{-2 h^{*}\left(1-\beta^{2}\right)}} \neq 0$. Since $k^{*} \neq 0$ these periodic orbits bifurcate from elliptic orbits $\left(g_{0} \neq 0\right)$ of the Kepler problem which are not in the plane of motion defined by the two bodies. This proves statement (b) of the theorem. 


\section{APPENDIX}

Now we shall present the basic results from averaging theory that we need for proving the results of this paper.

The next theorem provides a first order approximation for the periodic solutions of a periodic differential system, for the proof see Theorems 11.5 and 11.6 of Verhulst [17].

Consider the differential equation

$$
\dot{x}=\varepsilon F(t, x)+\varepsilon^{2} R(t, x, \varepsilon), x(0)=x_{0},
$$

with $x \in D$ where $D$ is an open subset of $\mathbb{R}^{n}$, and $t \geq 0$. Moreover we assume that $F(t, \mathrm{x})$ is $T$ periodic in $t$. Separately we consider in $D$ the averaged differential equation

$$
\dot{y}=\varepsilon f(y), y(0)=x_{0},
$$

where

$$
f(y)=\frac{1}{T} \int_{0}^{T} F(t, y) d t
$$

Under certain conditions, see the next theorem, equilibrium solutions of the averaged equation turn out to correspond with $T$-periodic solutions of equation (16).

Theorem 8. Consider the two initial value problems (15) and (16). Suppose:

(i) $F$, its Jacobian $\partial F / \partial x$, its Hessian $\partial^{2} F / \partial x^{2}$ are defined, continuous and bounded by an independent constant $\varepsilon$ in $[0, \infty) \times D$ and $\varepsilon \in\left(0, \varepsilon_{0}\right]$.

(ii) $F$ is $T$-periodic in $t$ ( $T$ independent of $\varepsilon$ ).

(iii) $y(t)$ belongs to $D$ on the interval of time $[0,1 / \varepsilon]$.

Then the following statements hold.

(a) For $t \in[0,1 / \varepsilon]$ we have that $x(t)-y(t)=O(\varepsilon)$, as $\varepsilon \rightarrow 0$.

(b) If $p$ is a equilibrium point of the averaged equation (16) and

$$
\left.\operatorname{det}\left(\frac{\partial f}{\partial y}\right)\right|_{\mathrm{y}=p} \neq 0
$$

then there exists a $T$-periodic solution $\varphi(t, \varepsilon)$ of equation $(15)$ such that $\varphi(0, \varepsilon) \rightarrow p$ as $\varepsilon \rightarrow 0$.

(c) The stability or instability of the periodic solution $\varphi(t, \varepsilon)$ is given by the stability or instability of the equilibrium point $p$ of the averaged system (16). In fact, the equilibrium point $p$ has the stability behavior of the Poincare map associated to the periodic solution $\varphi(t, \varepsilon)$.

\section{ACKNOWLEDGEMENTS}

We appreciate very much the comments of the reviewer which help us to improve the presentation of our results.

The first author was partially supported by MINECO/FEDER grant number MTM2011-22587, Junta de Comunidades de Castilla-La Mancha, grant 
number PEII09-0220-0222. The second author was partially supported by MICINN/FEDER grant number MTM2008-03437, AGAUR grant number 2009 SGR 410, ICREA Academia and FP7-PEOPLE-2012-IRSES-316338 and 318999. The third author was partially supported by Fundación Séneca de la Región de Murcia grant number 12001/PI/09.

\section{REFERENCES}

[1] Y. Alhassid, E.A. Hinds And D. Meschede, Dynamical symmetries of the perturbed hydrogen atom: The van der Waals interaction, Physical Review Letters 59 (1987), $1545-1548$.

[2] J. Casasayas, and J. Llibre, Qualitative analysis of the anisotropic Kepler problem, Mem. Amer. Math. Soc. 52, no. 312, (1984).

[3] G. Contopoulos And M. Harsoula, Stability and instability in the anisotropic Kepler problem, Journal Physical A 38 (2005), 8897-8920.

[4] B. Cordani, The Kepler problem, Progress in Mathematical Physics 29, SpringerVerlag, 2003.

[5] D. Farrelly and T. Uzer, Normalization and Detection of the Integrability: The Generalized van der Waals Potential Celestial Mechanics and Dynamical Astronomy 61 (1995), 71-95.

[6] J.L.G. Guirao, J. Llibre and J.A. Vera, Generalized van der Waals Hamiltonian: Periodic orbits and $C^{1}$ nonintegrability, Physical Review E 85 (2012), 036603.

[7] M. Gutzwiller And C. Martin, The quantization of a classically ergodic system. Classical quantum models and arithmetic problems, 287-351, Lecture Notes in Pure and Appl. Math. 92, Dekker, New York, 1984.

[8] R.C. Howison, K.R. Meyer, Doubly-symmetric periodic solutions of the spatial restricted three-body problem, J. Differential Equations 163 (2000), 174-197

[9] M. Iñarrea, V. Lanchares, J. Palacián, A.I. Pascual, J.P. Salas, P. Yanguas, Symplectic coordinates on $S^{2} \times S^{2}$ for perturbed Keplerian problems: application to the dynamics of a generalised Stormer problem, J. Differential Equations 250 (2011), 1386 1407.

[10] A. Maciejewski And H. Pretka, Galactic disc tidal action and observability of the Oort cloud comets, Astronomy and Astrophysics 336 (1998), 1065-1071.

[11] J. J. Matese And P. G. Whitman, The galactic disk tidal field and the nonrandom distribution of observed Oort cloud comets, Icarus 82 (1989), 389-401.

[12] J. J. Matese And P. G. Whitman, A model of the galactic tidal interaction with the Oort comet cloud, Celest. Mech. Dynam. Astron. 54 (1992), 13-35.

[13] K.R. Meyer, G.R. Hall and D. OfFin, Introduction to Hamiltonian dynamical systems and the N-body problem, Applied Mathematical Sciences 90, Springer New York, 2009.

[14] J. H. OORT. The structure of the cloud of comets surrounding the Solar System and a hypothesis concerning its origin, Bull. Astron. Inst. Neth. 11 (1950), 91-110.

[15] C. Robinson, An introduction to dynamical systemsÜcontinuous and discrete, Second edition. Pure and Applied Undergraduate Texts 19. American Mathematical Society, Providence, RI, 2012.

[16] M. SAntopRete. Block regularization of the Kepler problem on surfaces of revolution with positive constant curvature, J. Differential Equations 247 (2009), 1043-1063.

[17] F. Verhulst, Nonlinear Differential Equations and Dynamical Systems, Universitext, Springer, 1991. 
${ }^{1}$ Departamento de Matemática Aplicada y Estadística. Universidad Politécnica de Cartagena, Hospital de Marina, 30203-Cartagena, Región de Murcia, SPAIN.-CORRESPONDING AUTHOR-

E-mail address: juan.garcia@upct.es

2 Departament de Matemàtiques. Universitat Autònoma de Barcelona, Bellaterra, 08193-Barcelona, Catalonia, Spain

E-mail address: jllibre@mat.uab.cat

3 Centro Universitario de la Defensa. Academia General del Aire. Universidad Politécnica de Cartagena, 30720-Santiago de la Ribera, Región de MurCia, Spain

E-mail address: juanantonio.vera@cud.upct.es 